\title{
PROPAGACIÓN CLONAL in vitro DE PIÑA (Ananas comosus L. Merr) VARIEDADES CHAMPAKA Y HAWAIANA
}

\author{
Silvia Gicela Saucedo Aguiar ${ }^{1}$, Luis Edmundo Ramos Gavilanes ${ }^{1}$, Edgar Varas Giler ${ }^{2}$ y Fred Carmigniani Castro ${ }^{2}$ \\ ${ }^{1}$ Unidad de Investigación Cientifica y Tecnológica, Universidad Técnica Estatal de Quevedo. Av. Walter Andrade. \\ Km 1.5 vía a Santo Domingo, C.P. 73. Quevedo. Los Ríos, Ecuador. silgisauce@yahoo.es. \\ ${ }^{2}$ Facultad de Ciencias Agrarias, Universidad Técnica Estatal de Quevedo. Quevedo, Los Ríos, Ecuador.
}

\section{RESUMEN}

Este trabajo fue realizado con la finalidad de establecer un protocolo para la propagación in vitro de piña (Ananas comosus (L) Merr), así como determinar las mejores concentraciones de reguladores de crecimiento en el establecimiento, multiplicación, enraizamiento y aclimatación ex vitro. Para el establecimiento de piña var. Champaka y Hawaiana la mejor concentración fue en un medio de cultivo conformado por la totalidad de las sales del MS suplementado con BAP (0.5 mg $\left.\mathrm{L}^{-1}\right)$; AIB $\left(1.0 \mathrm{mg} \mathrm{L}^{-1}\right)$ y ANA $\left(1.0 \mathrm{mg} \mathrm{L}^{-1}\right)$ obteniéndose el 47 y $56 \%$ de supervivencia respectivamente. Para la proliferación de brotes la mejor concentración fue en un medio de cultivo conformado por la totalidad de las sales del MS suplementado con 3.5 y $4.0 \mathrm{mg} \mathrm{L}^{-1}$ de BAP en la var. Champaka y la var. Hawaiana con $3.5 \mathrm{mg} \mathrm{L}^{-1}$ de BAP. Para el enraizamiento ex vitro y aclimatación la mejor respuesta al número de raíces se presentó en el testigo (sin hormona) var. hawaiana con un promedio de 7.25. La mayor longitud se evidenció en el testigo (sin hormona) con $3.55 \mathrm{~cm}$ se obtuvo una sobrevivencia de $93.75 \%$ para la var. Hawaiana y 79.16 \% para la var. Champaka. Los mejores resultados en el enraizamiento y aclimataciones ex vitro de vitroplantas de piña se obtuvieron con el testigo en la variedad Champaka y con la auxina ANA (ácido naptalenacetico) $50 \mathrm{mg}$ $\mathrm{L}^{-1}$ en la var. Hawaiana.

Palabras clave: In vitro, reguladores de crecimiento, explantes, hormona.

\begin{abstract}
This work was realized by the purpose of establishing a protocol for the spread in vitro of pineapple (Ananas comosus (L) Merr), as well as to determine the best concentrations of regulators of growth in the establishment, multiplication, to take root and acclimatization ex vitro. For the establishment of pineapple var. Champaka and Hawaiian the best concentration was in a way of culture shaped by the totality of you them go out of the MS suplement with BAP $\left(0.5 \mathrm{mg} \mathrm{L}^{-1}\right)$; AIB $\left(1.0 \mathrm{mg} \mathrm{L}^{-1}\right)$ and ANA (1.0 $\left.\mathrm{mg} \mathrm{L}^{-1}\right) 47$ and $56 \%$ of survival being obtained respectively. For the proliferation of outbreaks the best concentration was in a way of culture shaped by the totality of you them go out of the MS suplement with 3.5 and $4.0 \mathrm{mg} \mathrm{L}^{-1}$ of BAP in the var. Champaka and the hawaiian var. with $3.5 \mathrm{mg} \mathrm{L}^{-1}$ of BAP. For the enraizamiento ex vitro and aclimatization the best response to the number of roots appeared in the witness (without hormone) var. Hawaiian with an average of 7.25. The major length was demonstrated in the witness (without hormone) by $3.55 \mathrm{~cm}$. There was obtained a survival of $93.75 \%$ for the Hawaiian var. and $79.16 \%$ for the var. champaka. The best results in the enraizamiento and acclimatizations ex vitro of vitroplantas of pineapple(pine cone) were obtained by the witness in the variety Champaka and by the auxina ANA (acid naptalenacetico) 50 $\mathrm{mg} \mathrm{L}^{-1}$ in the Hawaiian var..
\end{abstract}

Key words: In vitro, regulators of growth, explantes, hormone.

\section{INTRODUCCIÓN}

La piña es una Bromeliaceae, la cual se propaga vegetativamente, presentando un porcentaje de multiplicación muy lento. En la actualidad ha adquirido gran importancia comercial a nivel mundial por la demanda de su fruto. Esto ha llevado a la búsqueda de nuevos métodos de propagación, que permitan acelerar la producción de propágulos para la siembra en el campo entre los cuales cabe señalar el cultivo "in vitro"

Recibido: Octubre, 2007. Aceptado: Enero: 2008.

Publicado como ARTÍCULO en Ciencia y Tecnología 1: 49-54. 2008. de yemas axilares, que permite mejorar sustancialmente el proceso de multiplicación (Casale y García, 1997). En el país existen aproximadamente 7,000 hectáreas sembradas de piña de exportación. Esta fruta ocupa la primera posición entre las exportaciones no tradicionales hortifrutícolas del Ecuador. Además de exportar piña fresca, también se exporta elaborados de piña en jugo/ concentrado y conserva. En 1999, se exportaron 2,045 t de jugo de piña y $385 \mathrm{t}$ de piña en conserva (CORPEI, 2000).

Para el agricultor nacional la piña representa un cultivo de alta rentabilidad, de fácil manejo y con 
buenas perspectivas comerciales. La existencia de un importante mercado interno, pero sobretodo, las posibilidades de industrialización y exportación, crean magnificas expectativas para este cultivo (Pacheco, 1984). La producción de plantas de piña a nivel nacional asciende a 369'960,000 u en el año 1999, las que alcanzaron a cubrir un total de 6,166 ha de las cuales 33,296,400 fueron plantas meristemáticas. La producción nacional de plantas meristemáticas de piña en el año 1999 asciende a 8,324,100, de las cuales la tercera parte de la demanda fueron importadas de Costa Rica, Colombia, entre otros.

Las variedades cultivadas en el ámbito mundial son: Cayena Lisa, Queen y Española. En el Ecuador las variedades más sembradas son la Hawaiana, la Nacional y Champaka F-153 (Biblioteca Practica Agrícola y Ganadera, 1987). En este trabajo se pretende establecer un protocolo para el establecimiento, multiplicación, $\mathrm{y}$ enraizamiento in vitro de yemas axilares de retoños de corona de Ananas comosus L. Merr, variedad Hawaiana y Champaka F-153.

\section{MATERIALES Y MÉTODOS}

\section{Establecimiento e inducción del crecimiento de explantes de piña}

El objetivo de esta etapa fue encontrar un medio de cultivo adecuado para el establecimiento in vitro de yemas axilares de piña var. Champaka y Hawaiana. La extracción y siembra de las yemas se realizó en la cámara de flujo laminar después de haber sido desinfectadas.

El medio de cultivo utilizado fue el MS (Murashige y Skoog, 1962) suplementado con thiamina $5 \mathrm{mg} \mathrm{L}^{-1}$; sulfato de adenina $40 \mathrm{mg} \mathrm{L}^{-1}$; myo-inositol $100 \mathrm{mg}$ $\mathrm{L}^{-1}$; sacarosa $30 \mathrm{~g} \mathrm{~L}^{-1}$; además; auxinas AIA, ANA, AIB en concentraciones de 1.0 a $2.0 \mathrm{mg} \mathrm{L}^{-1}$ y el BAP en concentraciones de 0.5 a $1.0 \mathrm{mg} \mathrm{L}^{-1}$. Los explantes, fueron sometidos a los siguientes tratamientos: 1) Champaka + MS + BAP (0.5 mg L $\left.\mathrm{m}^{-1}\right)$; AIB (1.0 $\left.\mathrm{mg} \mathrm{L}^{-1}\right)$ y ANA (1.0 mg L $\left.\mathrm{m}^{-1}\right) ; 2$ ) Champaka + MS + BAP (1.0 mg $\left.\mathrm{L}^{-1}\right)$; AIB (2.0 mg L $\left.{ }^{-1}\right)$ y ANA $\left.\left(2.0 \mathrm{mg} \mathrm{L}^{-1}\right) ; 3\right)$ Hawaiana $+\mathrm{MS}+\mathrm{BAP}\left(0.5 \mathrm{mg} \mathrm{L}^{-1}\right) ; \operatorname{AIB}\left(1.0 \mathrm{mg} \mathrm{L}^{-1}\right)$ y ANA $(1.0$ $\left.\mathrm{mg} \mathrm{L}^{-1}\right)$; 4) Hawaiana + MS + BAP (1.0 $\left.\mathrm{mg} \mathrm{L}^{-1}\right)$; AIB (2.0 $\left.\mathrm{mg} \mathrm{L}^{-1}\right)$ y ANA $\left(2.0 \mathrm{mg} \mathrm{L}^{-1}\right)$.

Se utilizó un diseño completamente al azar con un arreglo factorial 2 (variedades) x 2 (medios de cultivo) con cuatro tratamientos y cuatro repeticiones (cada unidad experimental estuvo conformada por ocho explantes). A los 21 días se realizó la evaluación de manera visual contando el número de explantes contaminados, fenolizados, quemados y la supervivencia.

\section{Multiplicación y elongación de los brotes}

El objetivo de esta fase fue evaluar el tipo y la concentración de la citoquinina a emplear en la multiplicación in vitro de piña var. Champaka $\mathrm{y}$ Hawaiana. Para realizar este experimento se utilizaron vitroplantas proveniente de la etapa de establecimiento, las mismas que fueron sometidas a los siguientes tratamientos: 1) Champaka + MS + BAP $\left(3.0 \mathrm{mg} \mathrm{L}^{-1}\right)+$ AIA (2 mg L $\left.\left.{ }^{-1}\right) ; 2\right)$ Champaka + MS + BAP (3.5 mg L $\left.{ }^{-1}\right)$ + AIA $\left.\left(2 \mathrm{mg} \mathrm{L}^{-1}\right) ; 3\right)$ Champaka + MS + BAP (4.0 mg $\left.\mathrm{L}^{-1}\right)+$ AIA $\left.\left(2 \mathrm{mg} \mathrm{L}^{-1}\right) ; 4\right)$ Hawaiana + MS + BAP (3.0 $\left.\left.\mathrm{mg} \mathrm{L} \mathrm{L}^{-1}\right)+\mathrm{AIA}\left(2 \mathrm{mg} \mathrm{L}^{-1}\right) ; 5\right)$ Hawaiana + MS + BAP (3.5 $\left.\mathrm{mg} \mathrm{L}^{-1}\right)+$ AIA $\left.\left(2 \mathrm{mg} \mathrm{L}^{-1}\right) ; 6\right)$ Hawaiana + MS + $\operatorname{BAP}\left(4.0 \mathrm{mg} \mathrm{L}^{-1}\right)+\operatorname{AIA}\left(2 \mathrm{mg} \mathrm{L}^{-1}\right)$.

Se utilizó un diseño completamente al azar con un arreglo factorial 2 (variedades) x 3 (concentraciones de BAP) con cuatro tratamientos y cuatro repeticiones (cada unidad experimental estuvo conformada por ocho explantes). A los 60 días se evaluó número de brotes por explante, longitud promedio de brotes y vigor.

\section{Enraizamiento y aclimatación ex vitro}

El objetivo en esta fase, fue la de determinar la mejor concentración de las auxinas AIB y ANA en el enraizamiento de las vitroplantas de las var. Champaka y Hawaiana. Se utilizaron vitroplantas de la etapa de multiplicación con una altura de cuatro a cinco centímetros, con 2 o 3 pares de hojas. Posteriormente fueron colocadas en un sustrato de arena bajo túnel de polietileno y con un riego de Microjet con una frecuencia de tres minutos cada tres horas. Las vitroplantas fueron sometidas a los siguientes tratamientos: 1) Champaka + AIB (50 mg L $\left.{ }^{-1}\right) ; 2$ ) Champaka + ANA (50 mg L-1); 3) Champaka Sin hormona; 4) Hawaiana + AIB (50 mg $\left.\mathrm{L}^{-1}\right)$; 5) Hawaiana + ANA (50 mg L $\left.\mathrm{m}^{-1}\right)$; 6) Hawaiana sin hormona.

Se dispuso de un diseño completamente al azar con un arreglo factorial 2 (variedades) x 3 (hormonas) con cuatro tratamientos y cuatro repeticiones (cada unidad experimental estuvo conformado por ocho explantes). A los 45 días se evaluó el porcentaje de enraizamiento, número de raíces por planta, longitud de raíces, vigor y supervivencia. En todas las fases de propagación se utilizó la prueba Tukey al nivel de 0.05 , para establecer la diferencia entre medias.

\section{RESULTADOS Y DISCUSIÓN}

Los resultados obtenidos durante el proceso de micropropagación de la piña se analizaron por etapas.

\section{Establecimiento e inducción del crecimiento}

En el Cuadro 1, se observa que la var. Champaka presenta el mayor valor promedio de contaminación 1.72, mientras que la Hawaiana presentó un valor promedio de 1.62 en las yemas establecidas; por lo que se consideró que el método de desinfección propuesto por Bravo (1997) y modificado en la concentración de 
hipoclorito de sodio del $1.0 \%$ al $0.1 \%$ dio resultados eficientes. Esto concuerda con lo manifestado por Okabe (1997), quien indica que el material proveniente del campo debe desinfectarse antes de introducirlo a condiciones asépticas. Por esta razón se recomienda introducir el material al invernadero y tratarlo en un período prudente con fungicidas para bajar la incidencia de patógenos endógenos.
En lo referente a explantes fenolizados se obtuvo un mayor promedio en la var. Champaka 1.09 y 0.90 en la Hawaiana; el medio de cultivo 2 (MS +BAP $1.0 \mathrm{mg} \mathrm{L}^{-1}+$ AIB $2.0 \mathrm{mg} \mathrm{L}^{-1}+$ ANA $2.0 \mathrm{mg} \mathrm{L}^{-1}$ ) fue el que presentó mayor fenolización posiblemente este resultado podría atribuirse al hecho de que el material vegetal utilizado provenía de la segunda generación de la planta, lo cual concuerda con lo manifestado por Ramos (2000), quien

Cuadro 1. Efectos simples de dos variedades de piña y dos medios de cultivo en la etapa de establecimiento

\begin{tabular}{|c|c|c|c|c|}
\hline \multirow{2}{*}{ VARIEDADES } & \multicolumn{4}{|c|}{ Explantes } \\
\hline & Contaminados & Fenolizados & Quemados & Vivos (\%) \\
\hline Champaka & $1.72 \mathrm{a}^{\dagger}$ & $1.09 \mathrm{a}^{\dagger}$ & $1.12 \mathrm{a}^{\dagger}$ & $46.88 \mathrm{a}^{\dagger}$ \\
\hline Hawaiana & $1.62 \mathrm{a}$ & $0.90 \mathrm{a}$ & $1.09 \mathrm{a}$ & $56.25 \mathrm{a}$ \\
\hline \multicolumn{5}{|l|}{ MEDIOS DE CULTIVO } \\
\hline $\begin{array}{l}\text { MS+BAP 0.5 mg L L }^{-1} \text { AIB } 1.0 \mathrm{mg} \mathrm{L}^{-1} \\
+ \text { ANA } 1.0 \mathrm{mg} \mathrm{L}^{-1}\end{array}$ & $1.80 \mathrm{a}$ & $0.90 \mathrm{a}$ & $0.90 \mathrm{a}$ & $54.69 a$ \\
\hline $\begin{array}{l}\text { MS+BAP } 1.0 \mathrm{mg} \mathrm{L}^{-1}+\text { AIB } 2.0 \mathrm{mg} \mathrm{L}^{-1} \\
+ \text { ANA } 2.0 \mathrm{mg} \mathrm{L}^{-1}\end{array}$ & $1.55 \mathrm{a}$ & $1.09 \mathrm{a}$ & $1.31 \mathrm{a}$ & $48.43 \mathrm{a}$ \\
\hline CV\% & 22.21 & 22.15 & 34.19 & 26.65 \\
\hline
\end{tabular}

Medias con la misma letra no presentan diferencias estadísticas (Tukey, $\mathrm{P}=0.05$ ).

indica que la síntesis de los precursores de fenoles es más activa y compleja en tejidos maduros que en los tejidos jóvenes y está directamente influenciado por el contenido de sales y reguladores de crecimiento en el medio de cultivo (Cuadro 1). La var. Hawaiana fue la que presentó un mayor porcentaje de explantes vivos con un promedio de 56.25 y en cuanto a los medios de cultivo el mejor fue el medio 1 para las dos variedades. En esta fase no se presentaron diferencias estadísticas para explantes contaminados, fenolizados, quemados $\mathrm{y}$ vivos, tanto para variedades como para medios de cultivo (Cuadro 1).

\section{Multiplicación y elongación de los brotes}

En esta fase, la var. Champaka presentó el mayor número de brotes por explante. Al respecto Escalona (1999), manifiesta que con la aplicación de medios líquidos en el cultivo de piña, se puede triplicar el coeficiente de multiplicación. En cambio, para longitud $\mathrm{y}$ vigor de los brotes no se encontraron diferencias estadísticas con respecto a la var. Hawaiana. Con la concentración de 3.5 y $4.0 \mathrm{mg} \mathrm{L}^{-1}$ de BAP se encontró el mayor número de brotes y con $3.0 \mathrm{mg} \mathrm{L}^{-1}$ de BAP la mayor longitud de brotes (Cuadro 2). Esto concuerda con lo manifestado por Daquinta (1998), quien menciona que con la aplicación de auxina al medio de multiplicación incide directamente en el número de brotes puesto que al haber un balance de citoquinina-auxina provoca que los brotes no se elonguen en forma desordenada y se inhiban el enraizamiento, estimulando la brotación.

De acuerdo a esto se puede observar que para la variable número de brotes en la significación de la interacción "variedad x concentración de BAP, la var. Champaka presentó su mejor comportamiento con 3.5 y $4.0 \mathrm{mg} \mathrm{L}^{-1}$ de BAP y, la var. Hawaiana con $3.5 \mathrm{mg} \mathrm{L}^{-1}$ de BAP (Figura 1).

Asimismo, para longitud del brote, la variedad Champaka presentó mejores resultados con $3.0 \mathrm{mg} \mathrm{L}^{-1}$ de BAP y, la var, Hawaiana con 3.0 y $3.5 \mathrm{mg} \mathrm{L}^{-1}$ de BAP (Figura 2).

\section{Enraizamiento y aclimatación ex vitro}

Las variedades de piña presentaron diferencias estadísticas para porcentaje de enraizamiento, longitud, vigor y porcentaje de sobrevivencia. La variedad Champaka solo presentó mayor porcentaje de raíces; en cambio, la var. Hawaiana presentó mayor número de raíces por planta, longitud de raíz, vigor y sobrevivencia (Cuadro 3). Esto concuerda con lo manifestado por Pérez (1998), quien manifiesta que en la etapa de enraizamiento, 
es importante obtener un mayor número de raíces, aún de poca longitud, ya que las plantas obtenidas de cultivo in vitro requieren de un buen sistema radicular (mayor número de raíces) para tener éxito en transplante y adaptación a condiciones de invernadero, a diferencia de otras especies que son propagadas convencionalmente en forma asexual y que no requieren de raíces o muy pocas para ser transplantadas a sustrato. En lo referente al porcentaje de enraizamiento y sobrevivencia se encontraron diferencias estadísticas para la hormona ANA que presentó mayores valores.

En relación a la interacción "variedad x tipo de hormona", se encontró significación para la misma en todas las variables estudiadas. Encontrándose una mejor respuesta a la aplicación de hormonas por parte de la var. Hawaiana, no así para la var. Champaka, cuya mejor respuesta es sin hormonas (Figuras 3 y 4). Esto concuerda con lo manifestado por Casale y García, (1987) quien manifiesta que cuando el enraizamiento ocurre en tratamientos sin reguladores, se considera que la auxina no es necesaria para el enraizamiento, ni crecimiento de las raíces de piña, lo cual también fue encontrado durante la propagación in vitro de los cultivares "Española Roja" y "Nacional". Esto difiere de lo manifestado por Fitchet-Purnell (1993) que han reportado buenos resultados con la aplicación de 1 mg. $\mathrm{L}^{-1}$ de ANA para inducir el enraizamiento en la piña.

Cuadro 2. Efectos simples de dos variedades de piña y tres concentraciones de BAP en la etapa de multiplicación

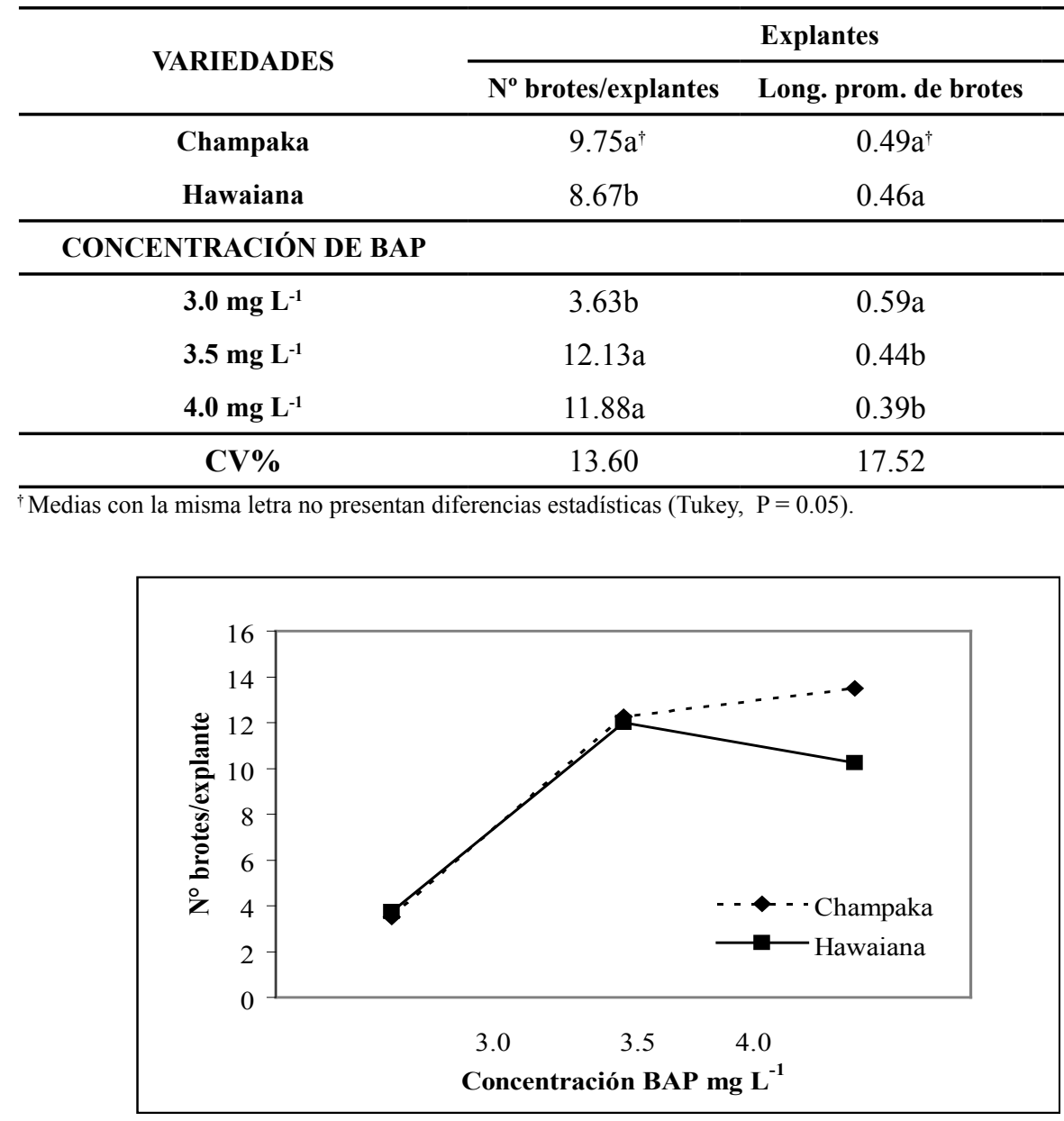

Figura 1. Interacción "variedad x concentración de BAP” en el número de brotes en la fase de multiplicación 


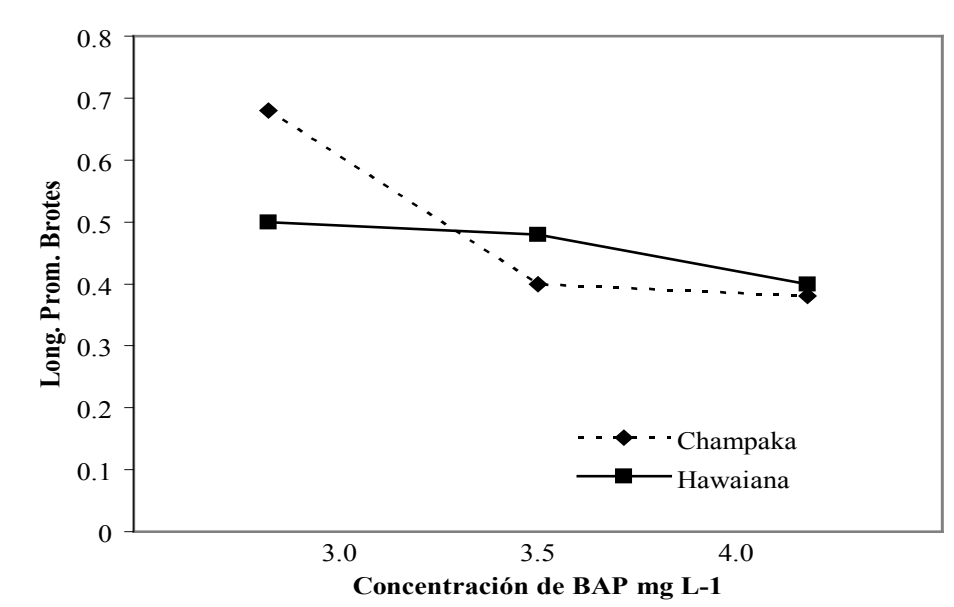

Figura 2. Interacción "variedad x concentración de BAP" en la longitud de brotes por explante en la fase de multiplicación

Cuadro 3. Efectos simples de dos variedades de piña y tres concentraciones de hormona en la etapa de enraizamiento

\begin{tabular}{cccccc}
\hline & \multicolumn{5}{c}{ Explantes } \\
\cline { 2 - 6 } VARIEDADES & $\begin{array}{c}\text { Enraizmiento } \\
\mathbf{( \% )}\end{array}$ & $\begin{array}{c}\text { \# de raíces } \\
\text { por planta }\end{array}$ & $\begin{array}{c}\text { Longitud } \\
\text { raíces (cm) }\end{array}$ & $\begin{array}{c}\text { Vigor } \\
(\text { Esc:1-3)*** }\end{array}$ & $\begin{array}{c}\text { Sobrevivencia } \\
(\%)\end{array}$ \\
\hline Champaka & $79.17 \mathrm{~b}^{\dagger}$ & $7.00 \mathrm{a}^{\dagger}$ & $2.26 \mathrm{~b}^{\dagger}$ & $2.08 \mathrm{~b}^{\dagger}$ & $79.16 \mathrm{~b}^{\dagger}$ \\
Hawaiana & $93.75 \mathrm{a}$ & $7.25 \mathrm{a}$ & $3.55 \mathrm{a}$ & $2.83 \mathrm{a}$ & $93.75 \mathrm{a}$ \\
\hline HORMONA & & & & & \\
\hline AIB** & $78.13 \mathrm{~b}$ & $6.88 \mathrm{a}$ & $2.64 \mathrm{a}$ & $2.50 \mathrm{a}$ & $78.13 \mathrm{~b}$ \\
ANA** & $92.19 \mathrm{a}$ & $7.88 \mathrm{a}$ & $2.78 \mathrm{a}$ & $2.25 \mathrm{a}$ & $92.19 \mathrm{a}$ \\
SIN HORMONA & $89.07 \mathrm{ab}$ & $6.63 \mathrm{a}$ & $3.30 \mathrm{a}$ & $2.63 \mathrm{a}$ & $89.06 \mathrm{ab}$ \\
\hline CV\% & 11.56 & 14.13 & 22.54 & 17.28 & 11.56
\end{tabular}

Medias con la misma letra no presentan diferencias estadísticas (Tukey, $\mathrm{P}=0.05$ ).

**AIB = Acido indolbutirico; ANA = Acido naftalenacético.

$* * * 1=$ Bajo; 2 = Medio; $3=$ Alto.

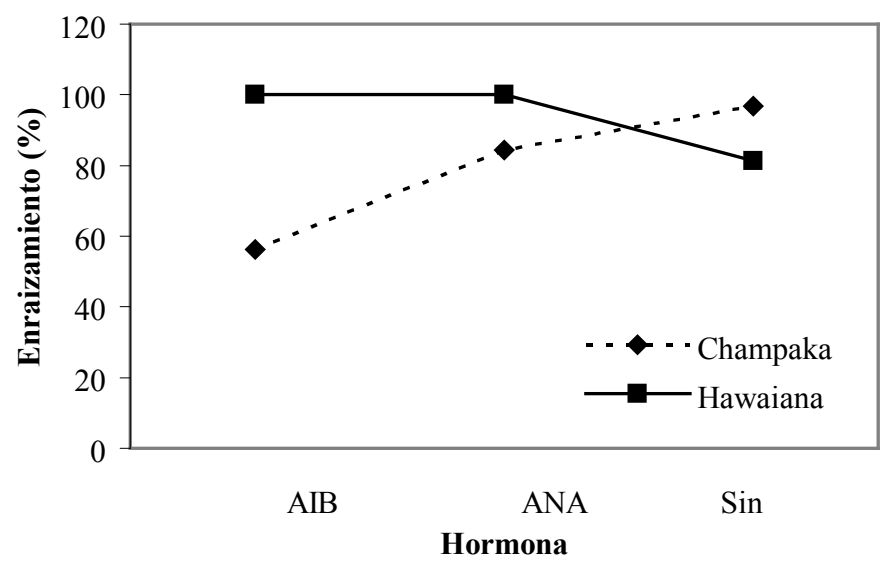

Figura 3. Interacción "variedad x tipo de hormona" en el porcentaje de enraizamiento en la fase de enraizamiento 


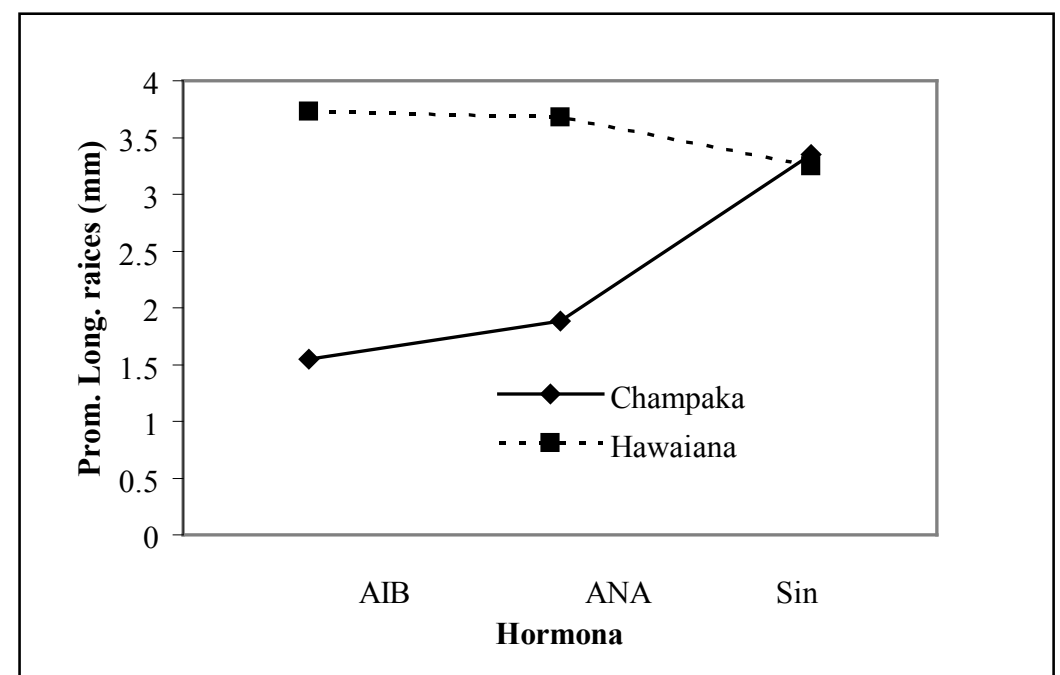

Figura 4. Interacción "variedad x tipo de hormona" en el promedio longitud de raíces en la fase de enraizamiento

\section{CONCLUSIONES}

En la fase de establecimiento el mejor medio de cultivo fue el MS suplementado con $0.5 \mathrm{mg} \mathrm{L}^{-1}$ de BAP $1.0 \mathrm{mg} \mathrm{L}^{-1}$ de AIB y $1.0 \mathrm{mg} \mathrm{L}^{-1}$ de ANA para las dos variedades (Champaka y Hawaiana). En la fase de multiplicación la variedad Champaka presentó mejor respuesta en el medio de cultivo suplementado con $3.5 \mathrm{mg} \mathrm{L}^{-1}$ de BAP y la variedad Hawaiana con la concentración de $4.0 \mathrm{mg} \mathrm{L}^{-1}$ de BAP. Se obtuvo un buen porcentaje de enraizamiento y supervivencia en la variedad Champaka sin la utilización de hormona y en la variedad Hawaiana con la hormona ANA $50 \mathrm{mg} \mathrm{L}^{-1}$.

\section{LITERATURA CITADA}

Biblioteca Práctica Agrícola y Ganadera. 1987. Práctica de los Cultivos. Oceano-Centrum. Tomo 2. Barcelona, España. p. 197 - 199.

Bravo L. 1997. Effects of benzylaminopurine (BAP) on in vitro proliferation of pineapple (Ananas comosus (L.) Merr.). Acta Hort. 425: 235-242

Casale, M. y García E, 1987. Multiplicación Clonal acelerada de tres variedades de piña, Centro de Botánica Tropical. Caracas, Venezuela. pp. 248 $-269$.

CORPORACION DE PROMOCION DE EXPORTACIONES E INVERSIONES. (CORPEI). 2000. Grandes expectativas por la piña de exportación. EL AGRO. (ECUADOR) $\mathrm{N}^{\circ} 50: 26-27$.
Daquinta, M. 1998. Propagación in vitro de la piña (Ananas comosus (L.) Merr.). Tesis presentada en opción al grado científico de Doctor en Ciencias Agrícolas. Universidad de Ciego de Avila, Cuba. 99p.

Escalona, M. 1998. Propagación in vitro de la piña (Ananas comosus (L.) Merr.) en sistema de inmersión temporal. Tesis para optar por el grado científico de Doctor en Ciencias Agrícolas. Universidad de Ciego de Avila, Cuba. 26p.

Fitchet-Purnell, M. 1993. Maximum utilization of pineapple crowns for micropropagation. Acta Hort. 334: 325-330.

Murashige, T. and Skoog. F. 1962. A revied medium for rapid growth and biossays with tobacco tissue culture. Plant physiol. 15: 173- 197.

Okabe, K. 1997. Principios básicos del cultivo de tejidos vegetales (volumen 2) Informe final. ICTA (Instituto de Ciencias y Tecnología Agrícola). Voluntarios Japoneses en Cooperación Técnica con el Extranjero (JOCV). Bárcena. Guatemala. p. 90.

Pacheco, M. 1984. El cultivo de la piña; Curso de Fruticultura (segunda parte). Facultad de Ciencias Agrícolas. Universidad Central de Ecuador. Quito, Ecuador. pp. 88 - 107.

Pérez Ponce, J.N. 1998. Propagación y Mejora Genética de Plantas por Biotecnología. Instituto de Biotecnología de las Plantas. Volumen I.

Ramos, L. 2000. Algunos avances en la morfogénesis de la teca (Tectona grandis L.) Universidad de Ciego de Avila. Centro de Bioplantas. Tesis para optar por el grado de Master en Ciencias. Santa Clara, Cuba. 55 p. 